\title{
Evaluación de la estrategia farmacoinvasiva versus angioplastia coronaria transluminal percutánea primaria en pacientes con infarto agudo de miocardio con elevación del segmento ST en el Instituto Nacional de Cardiología (PHASE-MX)
}

\section{Evaluation of pharmacoinvasive strategy versus percutaneous coronary intervention in patients with acute myocardial infarction with ST segment elevation at the National Institute of Cardiology (PHASE-MX)}

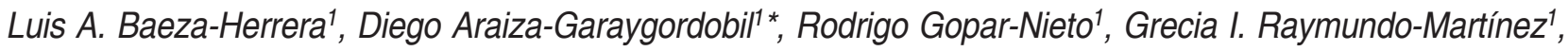
Arnoldo Loáisiga-Sáenz' ${ }^{1}$, Abraham Villalobos-Flores ${ }^{1}$, Manuel Martínez-Ramos ${ }^{1}$, Laura V. Torres-Araujo ${ }^{1}$, Ricardo Pohls-Vázquez ${ }^{1}$, Abraham Luna-Herbert', Arturo Alonso-Vázquez'1 Itzel V. Delgado-Cruz', Alejandro Cabello-López², Pablo Martínez-Amezcua ${ }^{3}$ y María A. Arias-Mendoza1

${ }^{1}$ Urgencias y Unidad de Cuidados Coronarios, Instituto Nacional de Cardiología Ignacio Chávez; ${ }^{2}$ Unidad de Investigación de Salud en el Trabajo, Centro Médico Nacional Siglo XXI, Instituto Mexicano del Seguro Social. Ciudad de México, México; ${ }^{3}$ Departmento de Epidemiología, Johns Hopkins Bloomberg School of Public Health, Baltimore, Maryland, EE.UU.

\begin{abstract}
Resumen
Objetivo: El objetivo del registro PHASE-MX es validar la eficacia y seguridad de la estrategia farmacoinvasiva en comparación con la angioplastia coronaria transluminal percutánea primaria (ACTPp) en pacientes con infarto agudo de miocardio con elevación del segmento ST (IAMCEST) en una región metropolitana de México. El desenlace primario es el compuesto de muerte cardiovascular, reinfarto, accidente vascular cerebral y choque cardiogénico. Métodos: El registro PHASE-MX es una cohorte prospectiva de pacientes con IAMCEST que recibieron tratamiento de reperfusión (mecánico o farmacológico) en las primeras 12 horas desde el inicio de los síntomas, atendidos en el Instituto Nacional de Cardiología Ignacio Chávez. El análisis estadístico se basa en la no inferioridad de la estrategia farmacoinvasiva en comparación con la ACTPp. Se calcula un tamaño de muestra de 344 pacientes divididos en dos grupos (angioplastia primaria y estrategia farmacoinvasiva), considerada una tasa de pérdidas de 10\%. Los pacientes incluidos en la cohorte PHASE-MX se seguirán durante un año. Discusión: En México, sólo 5 de cada 10 pacientes con IAMCEST tienen acceso al tratamiento de reperfusión. La estrategia farmacoinvasiva aprovecha la accesibilidad de la fibrinólisis y la efectividad de la ACTPp, por lo que podría resultar el método de elección en el tratamiento del IAMCEST en la mayoría de los casos. El presente protocolo de investigación pretende aportar información que sirva como enlace entre la información derivada de los estudios clínicos controlados y los registros derivados de la experiencia del mundo real.
\end{abstract}

Palabras clave: Infarto agudo de miocardio. Farmacoinvasivo. Reperfusión. México.

Correspondencia:

*Diego Araiza-Garaygordobil

E-mail: dargaray@gmail.com
Disponible en internet: 23-01-2020 Arch Cardiol Mex. 2020;90(2):158-162 www.archivoscardiologia.com 1405-9940/@ 2019 Instituto Nacional de Cardiología Ignacio Chávez. Publicado por Permanyer. Este es un artículo open access bajo la licencia CC BY-NC-ND (http://creativecommons.org/licenses/by-nc-nd/4.0/). 


\section{Abstract}

Objective: The objective of PHASE-MX registry is to validate the efficacy and safety of the pharmacoinvasive strategy in comparison with percutaneous coronary intervention $(P C I)$ in patients with acute myocardial infarction with ST segment elevation (STEMI) in a metropolitan region of Mexico. The primary outcome will consist of the composite of cardiovascular death, re-infarction, stroke and cardiogenic shock. Methods: The PHASE-MX registry will include a prospective cohort of patients with STEMI who received reperfusion treatment (mechanical of pharmacological) in the first $12 \mathrm{~h}$ after the onset of symptoms. The registry is designed to compare the efficacy and safety of primary PCl and pharmacoinvasive strategy. The simple size was calculated in 344 patients divided into two groups, with an estimated loss rate of $10 \%$. Patients included in the PHASE-MX cohort will be followed for up to one year. Conclusion: In Mexico, only 5 out of 10 patients with STEMI have access to reperfusion therapy. Pharmacoinvasive strategy is takes advantage of the accessibility of fibrinolysis and the effectiveness of $\mathrm{PCl}$. The present research protocol aims to provide information that serves as a link between information derived from controlled clinical trials and records derived from real world experience.

Key words: Acute myocardial infarction. Pharmacoinvasive. Reperfusion. Mexico.

\section{Introducción}

México es el país con la tasa de mortalidad de infarto agudo de miocardio con elevación del segmento ST (IAMCEST) más alta entre los países miembros de la Organización para el Desarrollo y la Cooperación Económica (OCDE), con un cálculo de 26.6 por cada 100 egresos, en comparación con el promedio de la OCDE de 8 muertes por cada 100 egresos ${ }^{1}$.

El tratamiento de reperfusión en el IAMCEST ha demostrado disminuir la morbimortalidad a largo pla$z^{2,3}$. Las guías de práctica clínica de la European Society of Cardiology recomiendan el uso de fibrinólisis 0 angioplastia coronaria transluminal percutánea primaria (ACTPp) con una clase de recomendación I, grado de evidencia $A$ en las primeras 12 horas tras el inicio de los síntomas ${ }^{4}$. Si bien la ACTPp resulta superior que la fibrinólisis al compararse de forma prospectiva en estudios clínicos aleatorizados y controlados ${ }^{3}$, los registros de la vida real sugieren que la fibrinólisis es un tratamiento eficaz cuando se administra en tiempo y previene retrasos relacionados con la angioplastia primaria ${ }^{5,6}$.

La estrategia farmacoinvasiva consiste en fibrinólisis inmediata y coronariografía sistemática subsecuente, preferentemente en las primeras 3 a 24 horas después de la fibrinólisis ${ }^{7}$. La ventaja de esta conducta permite el fácil acceso al fibrinolítico ${ }^{8}$ combinada con la eficacia de la angioplastia coronaria transluminal percutánea para prevenir el reinfarto y la isquemia recurrente ${ }^{3}$.La estrategia farmacoinvasiva ha demostrado no ser inferior en estudios clínicos prospectivos ${ }^{7,9}$, y registros de la vida real ${ }^{10}$, y múltiples países han publicado su experiencia en relación con sistemas de farmacoinvasión ${ }^{11}$.

La estrategia farmacoinvasiva podría resultar una forma viable en países y regiones donde el acceso a
ACTPp es limitada por razones geográficas, culturales o sociales, como México. Sin embargo, la estrategia farmacoinvasiva no se ha validado de forma prospectiva en pacientes mexicanos con IAMCEST.

El objetivo del estudio PHASE-MX es validar la eficacia y seguridad de la estrategia farmacoinvasiva en comparación con la angioplastia primaria en pacientes con IAMCEST en una región metropolitana de México.

\section{Metodología}

PHASE-MX es un estudio prospectivo de cohorte de pacientes con infarto agudo de miocardio con elevación del segmento ST tratados con fibrinólisis o angioplastia primaria y atendidos al final en un centro de tercer nivel en la Ciudad de México. El proyecto es una iniciativa de la Unidad de Urgencias y Unidad de Cuidados Coronarios del Instituto Nacional de Cardiología Ignacio Chávez y consiste en un registro prospectivo de todos los casos de IAMCEST que recibieron tratamiento de reperfusión en las primeras 12 horas. El seguimiento se extenderá hasta por 12 meses a partir del alta hospitalaria.

\section{Población de estudio}

Se incluirá a pacientes mayores de 18 años con diagnóstico de IAMCEST atendidos en alguno de los hospitales pertenecientes a la zona metropolitana de la Ciudad de México, sometidos a tratamiento de reperfusión (farmacológico o mecánico) en las primeras 12 horas desde el inicio de síntomas y referidos al Instituto Nacional de Cardiología Ignacio Chávez. El área de atención y referencia de la zona metropolitana de la Ciudad de México comprende un área calculada de $7,954 \mathrm{~km}^{2}$, con una población de 20.4 millones de 


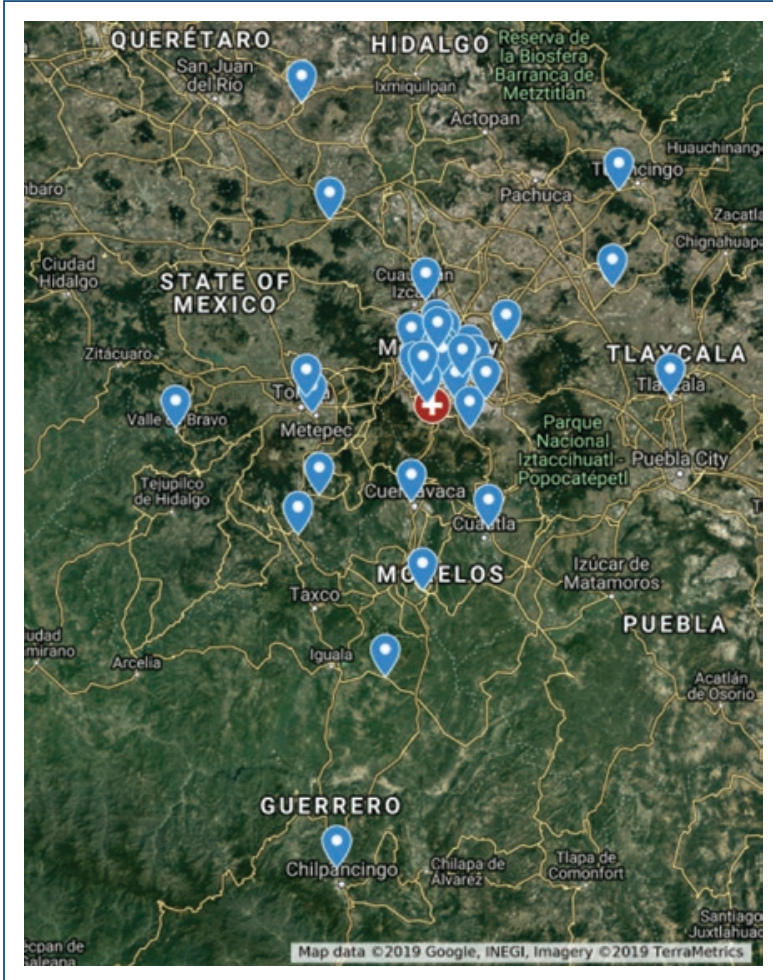

Figura 1. Mapa satelital que muestra el área de atención del Instituto Nacional de Cardiología Ignacio Chávez (INCICh). Los indicadores azules señalan la localización de los hospitales en donde los pacientes con IAMCEST recibieron tratamiento de reperfusión mediante fibrinólisis farmacológica, y luego fueron referidos al INCICh (marcado en el mapa con una cruz blanca sobre fondo rojo), durante un período aproximado de un año. El área metropolitana del Valle de México y el área de atención del INCICh tienen un área de extensión aproximada de 8,000 y $36,000 \mathrm{~km}^{2}$, respectivamente.

habitantes (Fig. 1 y Tabla 1) ${ }^{12}$. No se incluirá a los pacientes cuyo diagnóstico al egreso hospitalario sea diferente del IAMCEST.

\section{Seguimiento}

A partir del momento de inclusión se realizará seguimiento clínico hasta el egreso hospitalario. Después, al completar 12 meses de seguimiento (contabilizado a partir del egreso hospitalario), se analizará el estado vital por medio de contacto telefónico.

\section{Objetivos}

El objetivo general del registro PHASE-MX será evaluar la eficacia de la estrategia farmacoinvasiva en comparación con la angioplastia coronaria transluminal percutánea primaria en pacientes con infarto agudo de miocardio con elevación del segmento ST en el desenlace primario combinado de muerte cardiovascular, reinfarto, accidente vascular cerebral y choque cardiogénico en el seguimiento intrahospitalario. Los objetivos específicos serán conocer la frecuencia de sangrado mayor, accidente vascular cerebral y mortalidad por todas las causas a los 12 meses de seguimiento.

\section{Cálculo del tamaño de la muestra}

El objetivo primario será comparar el desenlace compuesto de EVC, reinfarto, choque cardiogénico y muerte cardiovascular, tras prever un poder estadístico (1- $\beta$ ) de $80 \%$, un nivel de $\alpha$ de 0.05 y tomar en cuenta una tasa prevista del desenlace primario de $12.4 \%$ en el seguimiento a 30 días de pacientes llevados a tratamiento de reperfusión y un grado máximo tolerado de diferencia (no inferioridad - d) de 5\%; se calcula una muestra total de 157 pacientes por brazo para demostrar no inferioridad de la estrategia farmacoinvasiva frente a la angioplastia primaria. Si se asume una tasa de pérdidas de $10 \%$, se calcula una muestra total final de 344 pacientes. La inscripción comenzó el 1 de abril de 2018 y su conclusión se espera para el 31 de marzo de 2020. Se prevé que la revisión y el análisis de la información estén disponibles para enviar a publicación a una revista de gran circulación en el segundo semestre de 2020.

\section{Análisis estadístico}

\section{Variables de estudio}

Además de las variables principales, se recabarán las siguientes:

- Infarto agudo de miocardio con elevación del segmento ST: pacientes con dolor torácico de características anginosas con un electrocardiograma de 12 derivaciones, con elevación del segmento ST. La elevación del segmento ST deberá ser en dos derivaciones contiguas, mayor de $2.5 \mathrm{~mm}$ en hombres menores de 40 años, mayor de $2 \mathrm{~mm}$ en hombres de más de 40 años o mayor de $1.5 \mathrm{~mm}$ en mujeres, en las derivaciones electrocardiográficas V2-V3 o mayor de $1 \mathrm{~mm}$ en cualquier otra derivación.

- Tiempo hasta el primer contacto médico: período de tiempo, medido en minutos desde el inicio de los síntomas indicativos de isquemia hasta recibir atención médica por primera vez. Ésta pueden proporcionarla un profesional de la salud o un técnico en urgencias médicas. 
Tabla 1. Hospitales por estado de la zona metropolitana de la Ciudad de México que refirieron a pacientes con IAMCEST al INCICh

\begin{tabular}{|l|c|c|}
\hline Ubicación de hospitales que refieren al INCICh & $\begin{array}{c}\text { Distancia promedio hasta el INCICh (km) } \\
\text { (mínimo-máximo) }\end{array}$ & $\begin{array}{c}\text { Tiempo promedio de transporte } \\
\text { (minutos)*(mínimo-máximo) }\end{array}$ \\
\hline Ciudad de México (15) & $21.6(12.5-31.8)$ \\
\hline Institutos Nacionales de Salud & $46.9(18-100)$ \\
\hline Estado de México (14) & $1.9(0.5-6.6)$ & $6(3-11)$ \\
\hline Total (34) & $88.8(29.6-190)$ \\
\hline
\end{tabular}

INCICh: Instituto Nacional de Cardiología Ignacio Chávez.

*Tiempo calculado mediante la aplicación Google Maps en horario 12:00-14:00 h, huso horario de la Ciudad de México.

**Incluye al Instituto Nacional de Ciencias Médicas y Nutrición Salvador Zubirán, Hospital General Dr. Manuel Gea González, Instituto Nacional de Enfermedades

Respiratorias Ismael Cossío Villegas, Instituto Nacional de Cancerología, Instituto Nacional de Neurología y Neurocirugía Dr. Manuel Velasco.

- Sitio de primer contacto médico: lugar en donde se llevó a cabo la atención médica inicial. Podrá corresponder a cualquier centro médico de primero, segundo o tercero niveles de atención.

- Tiempo puerta-aguja: período de tiempo medido en minutos, desde el momento de ingreso al sitio de primer contacto médico hasta el momento de recibir el fármaco fibrinolítico.

- Tiempo puerta-balón: lapso de tiempo medido en minutos, desde el momento de ingreso al sitio de atención médica con capacidad de ACTPp hasta el avance de la guía metálica más allá de la lesión en la arteria causante del infarto.

- Tiempo total de isquemia: tiempo transcurrido, medido en minutos, desde el inicio de los síntomas indicativos de isquemia hasta el momento de avance de la guía metálica más allá de la lesión en la ARI. En el caso de recibir fibrinólisis, se medirá desde el inicio de los síntomas hasta tener evidencia de reperfusión por medio del ECG, después de los 90 minutos de administrado el tratamiento fibrinolítico.

\section{Criterios de efectividad}

Angiográficos: flujo anterógrado en la ARI grado TIMI 3 y perfusión miocárdica TMP 3 .

Electrocardiográficos: descenso del segmento ST de al menos el $50 \%$ en relación con el electrocardiograma inicial, 90 minutos después de administrar el agente fibrinolítico.

\section{Desenlaces primarios}

- Desenlace primario combinado: muerte cardiovascular, choque cardiogénico, accidente vascular cerebral, reinfarto y choque cardiogénico en el seguimiento intrahospitalario.

- Mortalidad intrahospitalaria: defunción durante la hospitalización índice, relacionada o no con el motivo del ingreso principal.

- Accidente vascular cerebral: pérdida de la función de alguna región cortical o subcortical, como consecuencia de isquemia, embolismo o hemorragia, documentada por tomografía computarizada o resonancia magnética de cráneo.

- Infarto miocárdico no letal: infarto agudo de miocardio con elevación del segmento ST, según la definición previa, con paciente egresado vivo al final de la atención hospitalaria.

\section{Desenlaces secundarios}

- Hemorragia mayor: hemorragia que lleve a la muerte, sangrado retroperitoneal, sangrado de sistema nervioso central (incluido el ojo), sangrado con repercusión hemodinámica, sangrado que requiera intervención quirúrgica, descompresión de un compartimiento cerrado, transfusión de uno o más concentrados eritrocitarios, descenso de hemoglobina mayor de $3 \mathrm{~g} / \mathrm{dl}$ o descenso de hematócrito mayor de $10 \%$ respecto de una determinación previa.

- Hemorragia menor: hematuria microscópica sin relación con traumatismo uretral, epistaxis que requiere taponamiento o intervención quirúrgica, sangrado de tubo digestivo, sangrado conjuntival, hematoma mayor de $5 \mathrm{~cm}$, sangrado que reduzca la hemoglobina menos de $3 \mathrm{~g} / \mathrm{dl}$.

- Flujos TIMI, documentados por angiografía coronaria invasiva y categorizados de la siguiente manera: TIMI 0 : ausencia total de flujo; TIMI 1: penetración parcial 
del medio de contraste, sin cruzar la lesión causal ni llenar la arteria coronaria distal; TIMI 2: penetración del medio de contraste más allá de la lesión causal, sin llenar la arteria coronaria o, en caso contrario, con mayor lentitud que las arterias coronarias normales; TIMI 3: flujo arterial coronario normal.

- Mortalidad a los 12 meses de seguimiento.

\section{Discusión}

El estudio PHASE-MX tiene como finalidad demostrar mediante un análisis observacional de la vida real (es decir, sin aleatorización ni control de variables) que la fibrinólisis y posterior ACTP en pacientes con IAMCEST no es inferior en eficacia en comparación con la ACTPp. Las fortalezas de este estudio radican en tres puntos; el primero se refiere a la ausencia de grupos de aleatorización y de control; la segunda a la administración de fibrinólisis en una amplia variedad de contextos clínicos, y la tercera a la realización de ACTPp en un centro de referencia nacional, que se considera de alto volumen en angioplastia coronaria. Como principales debilidades de este estudio figuran la probabilidad de que los pacientes no sean trasladados al centro de inclusión por la gravedad de su condición clínica y la amplia variedad del tratamiento coadyuvante.

Una de las principales implicaciones derivadas de los hallazgos de este estudio es fundamentar el uso extendido de la fibrinólisis en México, en donde la disponibilidad de laboratorios de hemodinámica es limitada. Además, podrá ser el sustento científico de campañas de promoción en la atención del infarto en México.

\section{Financiamiento}

Los autores no recibieron patrocinio alguno para la realización de este protocolo. Los autores cubrieron todos los gastos generados.

\section{Conflicto de intereses}

Los autores declaran no tener ningún conflicto de intereses.

\section{Responsabilidades éticas}

Protección de personas y animales. Los autores declaran que para esta investigación no se han realizado experimentos en seres humanos ni en animales.

Confidencialidad de los datos. Los autores declaran que han seguido los protocolos de su centro de trabajo sobre la publicación de datos de pacientes.

Derecho a la privacidad y consentimiento informado. Los autores declaran que en este artículo no aparecen datos de pacientes.

Este protocolo de investigación se sometió al Comité de Ética e Investigación del Instituto Nacional de Cardiología Ignacio Chávez, con número de registro PT-19-109. Además, se registró en la plataforma ClinicalTrials.gov con el identificador NCT03974581.

\section{Bibliografía}

1. Secretaría de Salud. La atención del infarto agudo al miocardio en México. Estudio de oferta y demanda y análisis económico ex ante de un programa nacional de reducción de la mortalidad por infarto agudo al miocardio. Ciudad de México, 2017.

2. GUSTO investigators. An international randomized trial comparing thrombolytic strategies for acute myocardial infarction. NEJM. 1993; 32(10):673-682.

3. Keeley EC, Boura JA, Grines CL. Primary angioplasty versus intravenous thrombolytic therapy for acute myocardial infarction: a quantitative review of 23 randomised trials. The Lancet. 2003;361(9351):13-20.

4. Agewall S, Camm J, Barón Esquivias G, Budts W, Carerj S, Casselman F et al. Guía ESC 2016 sobre el diagnóstico y tratamiento de la fibrilación auricular, desarrollada en colaboración con la EACTS. Rev Esp Cardiol. 2017;70(1):50.1-50.e84.

5. Andersen HR, Kelbaek H, Madsen JK, Lomholt P, Mortensen LS. A comparison of coronary angioplasty with fibrinolytic therapy in acute myocardial infarction. N Engl J Med. 2003;349 (8):733-742.

6. Bonnefoy E, Steg PG, Boutitie F, Dubien P-Y, Lapostolle F, Roncalli J, et al. Comparison of primary angioplasty and pre-hospital fibrinolysis in acute myocardial infarction (CAPTIM) trial: a 5-year follow-up. Eur Heart J. 2009;30(13):1598-606.

7. Armstrong $P W$, Gershlick $A H$, Goldstein $P$, Wilcox R, Danays $T$, Lambert $\mathrm{Y}$, et al. Fibrinolysis or primary $\mathrm{PCl}$ in ST-segment elevation myocardial infarction. N Engl J Med. 2013;368(15):1379-87.

8. Steg PG, Bonnefoy E, Chabaud S. Impact of time to treatment on mortality after prehospital fibrinolysis or primary angioplasty: data from the captim randomized clinical trial. ACC Curr J Rev. 2004;13(3):56-7.

9. Pu J, Ding S, Ge H, Han Y, Guo J, Lin R, et al. Efficacy and safety of a pharmaco-invasive strategy with half-dose alteplase versus primary angioplasty in ST-segment-elevation myocardial infarction: EARLY-MYO Trial (Early Routine Catheterization After Alteplase Fibrinolysis Versus Primary PCl in Acute ST-Segment-Elevation Myocardial Infarction). Circulation. 2017:136(16):1462-73.

10. Fernández-Avilés F, Alonso JJ, Pena G, Blanco J, Alonso-Briales J, López-Mesa J, et al. Primary angioplasty vs. early routine post-fibrinolysis angioplasty for acute myocardial infarction with ST-segment elevation: the GRACIA-2 non-inferiority, randomized, controlled trial. Eur Heart J. 2007;28(8):949-60

11. Araiza-Garaygordobil D, Arias-Mendoza A, Martínez-Sánchez C Martínez-Ríos MA, Ajit MS. Reperfusion of myocardial infarction in India: notions for México. Arch Cardiol México. 2018;88(2):148-52.

12. Instituto Nacional de Estadística y Geografía. Censo Nacional de Población y Vivienda 2010. 1era ed. Ciudad de México, México. 2011. 